\title{
Cleaning Fabricated Metal Thread: A Post-treatment Stability Assessment after Artificial Deterioration and the Application of Synthetic Soil
}

\author{
Hae Jin Park | Minsun Hwang ${ }^{1}$ | Yong Jae Chung* \\ Department of Textile Conservation, The Metropolitan Museum of Art, New York, 10028, United States \\ * Department of Heritage Conservation and Restoration, Korea National University of \\ Cultural Heritage, Buyeo, 33115, Korea \\ ${ }^{1}$ Corresponding Author: MinSun.Hwang@metmuseum.org, +1-212-650-2465
}

\begin{abstract}
To study the cleaning effects and post-treatment stability assessment of various methods of cleaning textiles with metal thread, six naturally-soiled historical textiles with metal thread were investigated at the Metropolitan Museum of Art, New York. Prior to the cleaning of fabricated gold, silver, and copper thread that had been glued onto a paper substrate, the artificial deterioration was carried out in a controlled environment with light(UV and daylight), and temperature and humidity factors which would weaken and damage the samples. A synthetic soil mixture was applied to the samples to imitate soil found on the historic and archaeological textiles with metal thread; the cleaning effect and post-treatment assessment were investigated by use of three textile cleaning methods: mechanical cleaning, wet cleaning, and solvent cleaning. While investigating the naturally-soiled textiles with metal thread, it was determined that the soil colors and sizes of contaminating particles of each textile were different due to the diversity of original environmental factors and conditions. After cleaning with kneaded rubber, Stoddard solvent, $n$-decane or $n$-hexane, a bright, clean effect was apparent. Kneaded rubber was successful in picking up both large and small particles, but its stickiness caused some of the metal leaf to peel off. Stoddard solvent produced a good cleaning effect, but after use of n-hexane and n-decane in the cleaning process, a white layer of residue remained on the textile's surface. Wet cleaning was not effective and the rapid humidity changes between wet and dry conditions caused the edges of the paper substrate to lose their original shape.
\end{abstract}

Key Words: Synthetic soil, Metal thread, Post-treatment, Textile cleaning, Stability assessment

\section{INTRODUCTION}

Soil is an undoubtedly complicated mixture of carbonaceous, organic and/or inorganic materials. Many textile conservators and curators have faced the soiling of metal thread as an unavoidable phenomenon, occurring inevitably with solid particulate matter as a dust and/or fine mineral particles from the external environment. The extent of soiling can be influenced by various and complex environmental factors that have been directly and externally related to burial site, temperature and humidity, fog, airborne pollutants, air movement and/or circulation, molecular size and specific shape of dirt particles, surface condition, chemical characteristics, and external electrostatic force as Van der Waal's forces or hydrogen bonding.

This subject has been studied and discussed in the past. Wolbers' research reported that native soils contain both water soluble and non-soluble components, as well as organic and inorganic materials. He proposed a model soiling mixture and evaluated its removal from the oil paint film under various conditions, to better characterize and understand the possible sorption and desorption mechanisms involved in the retention of native soils(Wolbers, 1992). In addition, Chi and Obendorf(1998) reported that soil on textiles often originates from the wearer's body or immediate environment, and although the cause of yellowing may differ in various cases, human sebum can be a major source of oily soil on textiles. The undesirable stain of the oily soil 
may be followed by oxidation resulting from heat, UV radiation, and/or an environmental catalyzing process. In another experiment, Rees used the bleached cotton textile fabric in a cloud chamber for investigation of the relationship between optical and gravimetric assessment of particulate soiling. In his experiment, he discovered that colloidal graphite in distilled water was an excellent material to use as artificially visual soiling; however, it was extremely difficult to extract from cotton for quantitative assessment. Instead, he used ferric oxinate ground in a ball mill as an alternative soiling material(Ree, 1962; Chi and Obendorf, 1998). Following published research on sampling methods, evaluation was conducted by wiping, vacuuming, and using an acoustic dust generator to deposit house dust and lead oxides containing metal, allergens, pesticides, or other contaminants on textiles, linoleum, and wood materials (Que Hee et al., 1985; Reynolds et al., 1997; Roger et al., 1999).

The oxidation of oily soil causes undesirable yellowing, whereas the accumulation of particulate soils usually results in gray surface discoloration. Cleaning is often a first step of a textile's conservation process; such work can enhance the condition of textiles with metal thread for their longterm preservation. Mechanical and chemical cleaning methods have been widely used to clean corrosion on metal thread. According to earlier established studies on the cleaning of metal thread, Toth reported that corrosion cannot be cleaned completely from textiles with metal thread by methods without damaging integral parts of the artwork. Silver chloride was removed successfully in some parts of embroideries with ethanol-soaked swabs, and layers of dust were removed with a micro-vacuum cleaner. However, the attempt to remove black silver sulphide corrosion with a pencil eraser was unsuccessful because the rubber eraser damaged the silk thread securing the thin metal wire(Toth, 2012). To remove the corrosion on the metal thread, Ahmed used different types of solvents such as acetone, carbon tetrachloride, and methanol by immersing a piece of cotton wrapped around a wooden stick into the solvent solution and making contact with the metal thread surface. He then wiped the metal thread and brushed away all corrosion products(Ahmed, 2014).
As with previously published research, textile conservators studied cleaning methods for metal thread because soil is a troublesome problem which reduces the textiles' aesthetic appearance; it produces noticeable discoloration and also acts as a catalyst for continuing deterioration. However, most published research has focused on the cleaning method and technique only for the corrosion of metal thread. Very rarely have there been studies on cleaning experiments with different types of simulated soil, not simply on corrosion or patina of metal thread.

This study investigates cleaning effects and post-treatment stability assessment of various textile cleaning methods used with metal thread. Six naturally soiled historical textiles with metal thread were investigated. Prior to the cleaning experiment, the artificial deterioration was executed in a controlled environment with light(UV and daylight), and temperature and humidity factors to weaken and damage the fabricated samples. The synthetic soil mixture was applied on the samples in order to imitate the soil of metal thread; subsequent cleaning effects and post-treatment assessment were investigated with various textile cleaning methods: mechanical cleaning, wet cleaning, and solvent cleaning.

\section{MATERIAL AND METHOD}

\subsection{Investigation of soiled textiles with metal thread at the Metropolitan Museum of Art}

To investigate the natural soil and dirt on soiled textiles containing metal thread, six examples were selected from collections at the Metropolitan Museum of Art(Table 1). This study/survey of Chinese textiles with metal thread includes various types of metal thread, colors and conditions of soil and dirt so that a variety of naturally produced soils and dirt on metal thread could be examined under the microscope(Axio Zoom, V16, ZEISS, Germany).

\subsection{Fabrication and artificial deterioration of metal thread samples}

For the fabrication of metal thread samples, three types 
of metal leaf were used: $24 \mathrm{~K}$ gold, silver, and copper purchased from Barnabas Blattgold Company. For the paper substrate, Korean mulberry papers were used with the basis weight of $50.34 \mathrm{~g} / \mathrm{m}^{2}$ and $0.15 \mathrm{~mm}$ in thickness, crafted at a typical traditional Korean paper store, Janggibang. Prior to the adhesive application, sizing treatment was applied to paper substrates with $3 \%(\mathrm{w} / \mathrm{w})$ ground hide glue and $0.2 \%(\mathrm{w} / \mathrm{w})$ alum, and $10 \%(\mathrm{w} / \mathrm{w})$ ground hide glue adhesive solutions were used to the sized paper substrate. After the fabrication process, the controlled environmental deterioration weakened and damaged the metal samples to a condition similar to that of historic and archaeological metal thread. The deterioration process of the metal thread described in environmental deterioration used with light(UV and daylight), and temperature and humidity, was followed by previously published research(Park et al, 2018). The light deterioration

Table 1. Investigated textile collections with metal thread at the Metropolitan Museum of Art

\begin{tabular}{|c|c|c|c|c|c|c|c|}
\hline No. & $\begin{array}{c}\text { Object name } \\
\text { (Accession No.) }\end{array}$ & Classification & Date & Culture & Medium & Dimensions & Digital image \\
\hline 1 & $\begin{array}{c}\text { Textile } \\
\text { fragments } \\
(1997.216 a, b)\end{array}$ & $\begin{array}{l}\text { Textiles-Embro } \\
\text { idered }\end{array}$ & $\begin{array}{c}\text { Liao } \\
\text { Dynasty } \\
(907-1125)\end{array}$ & China & $\begin{array}{l}\text { Silk and metal } \\
\text { thread }\end{array}$ & $\begin{array}{c}13 \times 201 / 2 \mathrm{in} . \\
(33.0 \times 52.1 \mathrm{~cm})\end{array}$ & \\
\hline 2 & $\begin{array}{l}\text { Cloth and } \\
\text { border } \\
\text { fragments } \\
(1998.114 \mathrm{a}-\mathrm{d})\end{array}$ & Textiles & $\begin{array}{l}\text { Yuan Dynasty } \\
(1271-1368)\end{array}$ & China & $\begin{array}{l}\text { Silk and metal } \\
\text { thread }\end{array}$ & $\begin{array}{l}\text { Overall } \\
81 / 4 \mathrm{in} . \\
(21 \mathrm{~cm})\end{array}$ & \\
\hline 3 & $\begin{array}{c}\text { Textile } \\
\text { fragment } \\
(1998.438)\end{array}$ & Textiles-Woven & $\begin{array}{l}\text { 13th-mid-14th } \\
\text { century }\end{array}$ & China & $\begin{array}{l}\text { Silk and metal } \\
\text { thread }\end{array}$ & $\begin{array}{c}\text { Wrap } \\
1911 / 16 \mathrm{in} . \\
(50 \mathrm{~cm}) \\
\text { Weft } \\
195 / 16 \mathrm{in} . \\
(49 \mathrm{~cm})\end{array}$ & \\
\hline 4 & $\begin{array}{c}\text { Decorative } \\
\text { pendant } \\
(1989.4)\end{array}$ & $\begin{array}{c}\text { Textiles-Embro } \\
\text { idered }\end{array}$ & $\begin{array}{l}\text { Ming Dynasty } \\
(1368-1644)\end{array}$ & China & $\begin{array}{l}\text { Silk and metal } \\
\text { thread } \\
\text { embroidery on } \\
\text { silk satin }\end{array}$ & $\begin{array}{l}251 / 2 \times 12 \text { in. } \\
(64.8 \times 30.5 \mathrm{~cm})\end{array}$ & \\
\hline 5 & $\begin{array}{l}\text { Rank badge } \\
(1998.154 .1)\end{array}$ & $\begin{array}{l}\text { Textiles-Embro } \\
\text { idered }\end{array}$ & $\begin{array}{l}\text { Ming Dynasty } \\
(1368-1644)\end{array}$ & China & $\begin{array}{l}\text { Silk and metal } \\
\text { thread } \\
\text { embroidery on } \\
\text { silk gauze }\end{array}$ & $\begin{array}{c}\text { Overall } \\
141 / 2 \times 14^{1 / 4} \mathrm{in} . \\
(36.8 \times 36.2 \mathrm{~cm})\end{array}$ & \\
\hline 6 & $\begin{array}{c}\text { Woman's } \\
\text { dragon robe } \\
(62.162)\end{array}$ & $\begin{array}{c}\text { Costumes-Tape } \\
\text { stries }\end{array}$ & $\begin{array}{c}\text { Qing Dynasty } \\
\text { (1644-1911); } \\
\text { Yongzheng } \\
\text { Period } \\
(1723-1735)\end{array}$ & China & $\begin{array}{l}\text { Silk and metal } \\
\text { thread }\end{array}$ & $\begin{array}{l}54 \times 71 \text { in. } \\
(137.16 \times \\
180.34 \mathrm{~cm})\end{array}$ & \\
\hline
\end{tabular}


experiment was conducted in a light box(SpectraLight II, Macbeth, China) under both UV and daylight for 756,000 lux-hours; and daily average temperature and humidity inside the light box were $30.58^{\circ} \mathrm{C}$ and $34.06 \%$ respectively. The temperature and humidity deterioration was carried out inside a humidity cabinet(LHL-112M, ESPEC CROP, Japan), set to $80^{\circ} \mathrm{C}$ and $30 \%$ humidity for 2 hours. Next, the temperature was reduced to $25^{\circ} \mathrm{C}$ and humidity was raised to $85 \%$. The humidity cabinet remained at these levels for 30days period. Fabrication materials and deterioration conditions before cleaning are listed in Table 2 .

\subsection{Synthetic soiling}

The deterioration in a controlled environment had insufficient soiling/staining on the surface of samples for cleaning stability assessment, quite unlike historic and archaeological conditions to which textiles with metal thread would be subjected(Park et al, 2018). In order to mimic these condition, to the surface of the metal thread samples we added synthetic soil mixture consisted of: carbon black, iron oxide, gelatin, soluble starch, cement, silica, lime, kaolin, peat moss, and mineral oil followed by previously established articles as seen in Table 3(Wolbers,

Table 2. Fabrication materials and deteriorated metal thread samples for cleaning

\begin{tabular}{|c|c|c|c|c|}
\hline \multirow{2}{*}{ No. } & \multicolumn{3}{|c|}{ Fabrication of the metal thread samples } & \multirow{2}{*}{$\begin{array}{l}\text { Environmental factor } \\
\text { for deterioration }\end{array}$} \\
\hline & Substrate & Adhesive & Metal leaf & \\
\hline 1 & \multirow{6}{*}{ Korean mulberry paper } & \multirow{6}{*}{ Ground hide glue } & \multirow{2}{*}{ Gold } & Light(UV+daylight) \\
\hline 2 & & & & Temperature+Humidity \\
\hline 3 & & & \multirow{2}{*}{ Silver } & Light(UV+daylight) \\
\hline 4 & & & & Temperature+Humidity \\
\hline 5 & & & \multirow{2}{*}{ Copper } & Light(UV+daylight) \\
\hline 6 & & & & Temperature+Humidity \\
\hline
\end{tabular}

Table 3. Synthetic soiling materials

\begin{tabular}{cccc}
\hline No. & Synthetic soiling materials & Amount & Sources \\
\hline 1 & Carbon black & $0.175 \mathrm{~g}$ & Jacquard Jac-JPX1640 Pearl Ex, USA \\
\hline 2 & Iron oxide & $0.05 \mathrm{~g}$ & Fisher Scientific, USA \\
\hline 3 & Gelatin & $0.95 \mathrm{~g}$ & Zint LLC, USA \\
\hline 4 & Soluble starch & $0.95 \mathrm{~g}$ & Fisher Scientific, India \\
\hline 5 & Cement(Portland type I) & $1.7 \mathrm{~g}$ & Hechinger's Inc., USA \\
\hline 6 & Silica & $0.175 \mathrm{~g}$ & Talas, USA \\
\hline 7 & Lime & $1.525 \mathrm{~g}$ & Lhoist North American of Virginia, Inc., USA \\
\hline 8 & Kaolin & $1.7 \mathrm{~g}$ & Allgoodies Boutique, Portugal \\
\hline 9 & Peat moss & $1.9 \mathrm{~g}$ & Sphagnum Peat Moss, Fisons Horticultural Supply Co., Canada \\
\hline 10 & Mineral oil & $8.0 \mathrm{~mL}$ & Vi-Jon Inc., USA \\
\hline
\end{tabular}

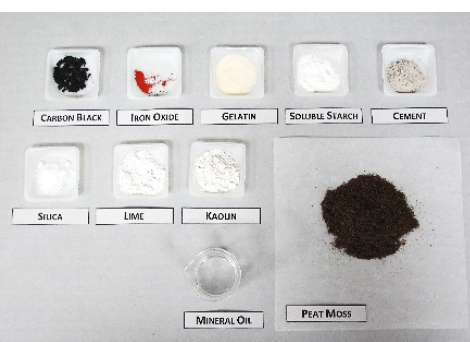

Figure 1. Synthetic soiling materials. Figure 2. Synthetic soiling mixture.

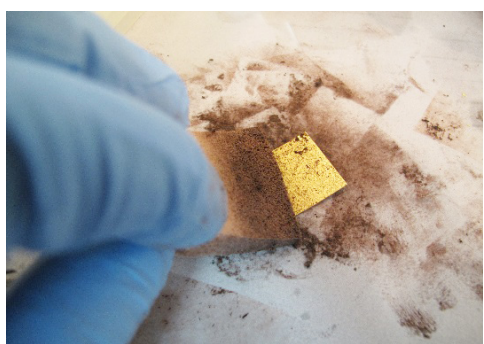

Figure 3. Application with sponge. 
1992). The synthetic soiling mixture consisted of a small amount of moist powder with various-sized particles, difficult to apply evenly over the samples' surface. We discovered that a $12.1 \mathrm{~g}$ drying mixture with $20 \mathrm{~mL}$ solution of $4.5 \%$ mineral oil in chloroform $(\mathrm{w} / \mathrm{w})$ created the slurry texture which could be applied more effectively to the metal samples' surface. We applied it evenly on the surface with no-residue sponges(Figures 1-3).

\subsection{Cleaning}

For the cleaning process, 11 different types of textile cleaning methods were applied to the artificial soil sitting on the fabricated metal samples following the deterioration shown in Table 4. First was the mechanical cleaning: brushing, vacuuming, and kneaded rubber. Brushing and vacuuming is well-known and used commonly as a first step of cleaning; kneaded rubber is used to pick up solid powder and/or fragments and particle. Second was wet cleaning with de-ionized water(DI water) immersion, de-ionized water suction, $5 \%$ saponin, and 3\% Orvus WA Paste. In this study, DI water was applied as a solvent alone with two methods: aggressive immersion and gentle soft penetration under the suction table. Saponin is a natural detergent, an extraction from a large family of ginseng; and Orvus WA Paste is a close $\mathrm{pH}$ neutral anionic synthetic surfactant and wetting agent with excellent detergency. The third method employed was the solvent cleaning with n-decane, n-hexane, acetone, ethyl alcohol and Stoddard solvent. Stoddard solvent is a distilled petroleum mixture of $\mathrm{C}_{7}-\mathrm{C}_{12}$ hydrocarbons, a dry cleaning agent.

To eliminate additional mechanical force during handling and/or cleaning, wet and solvent cleanings were conducted with the appropriate suction force and solubility of each cleaning material(Figures 4, 5). The surface was microscopically examined before and after cleaning treatments(Axio Zoom, V16, ZEISS, Germany) and color change was recorded with a spectrophotometer(CM-2600d, KONICA MINOLTA, Japan).

Table 4. Various cleaning method of metal thread samples

\begin{tabular}{|c|c|c|c|}
\hline No. & & Cleaning methods & Sources \\
\hline 1 & Control & Non-cleaning & - \\
\hline 2 & \multirow{2}{*}{$\begin{array}{l}\text { Mechanical } \\
\text { cleaning }\end{array}$} & Brushing and vacuuming & - \\
\hline 3 & & Kneaded rubber & Talas, USA \\
\hline 4 & \multirow{4}{*}{ Wet cleaning } & De-ionized water immersion & - \\
\hline 5 & & De-ionized suction & - \\
\hline 6 & & 5\% Saponin / De-ionized water (w/w) & Junsei, Japan \\
\hline 7 & & $3 \%$ Orvus WA Paste / De-ionized water (w/w) & Procter\&Gamble, USA \\
\hline 8 & \multirow{5}{*}{ Solvent cleaning } & n-decane & AlfaAesar, USA \\
\hline 9 & & n-hexane & Acros Organics, USA \\
\hline 10 & & Acetone & Fisher Scientific Chemicals, Inc., USA \\
\hline 11 & & Ethyl alcohol & Fisher Scientific Chemicals, Inc., USA \\
\hline 12 & & Stoddard solvent & Fisher Scientific Chemicals, Inc., USA \\
\hline
\end{tabular}
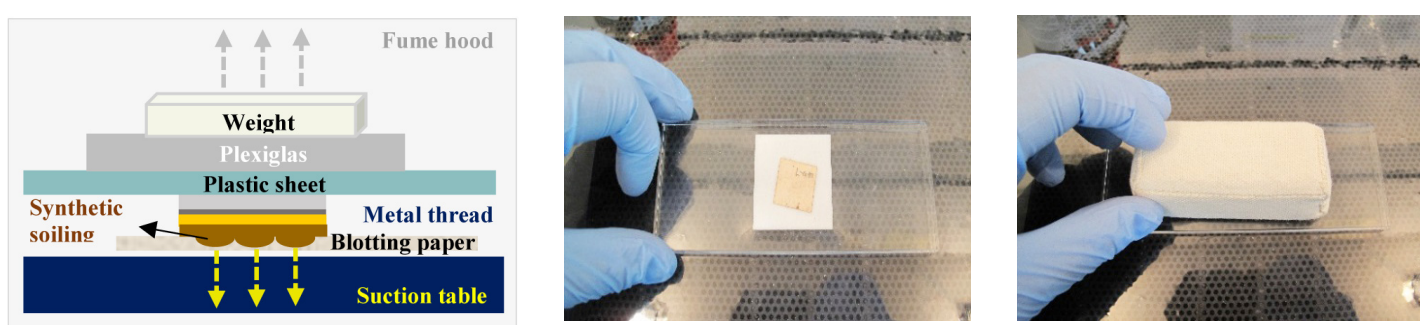

Figure 4. Setup for the wet and solvent cleaning. Figure 5. Wet and solvent cleaning experiment steps. 


\section{RESULTS}

3.1. Investigation of soiled textiles with metal thread at the Metropolitan Museum of Art

While investigating naturally-soiled textiles, we discovered that the effects of soiling were different because of the textile's particular preservation environments which clearly varied for each textile; the colors and size of particles varied as well. An tiger-dragon embroidery, dating to the Liao dynasty(907-1125), is an embroidered textile with

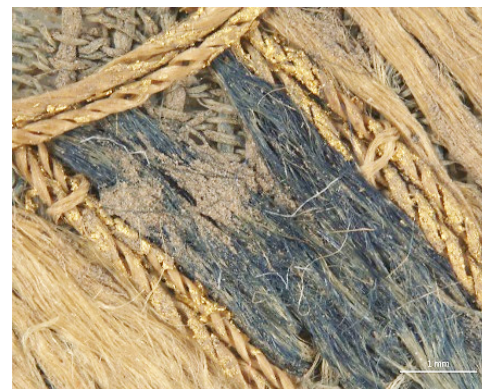

Figure 6. Natural soil on metal foil without organic substrate around silk core $(\times 20)$, embroidery with tiger- dragon, Liao Dynasty, China, 1997. 216a, b.

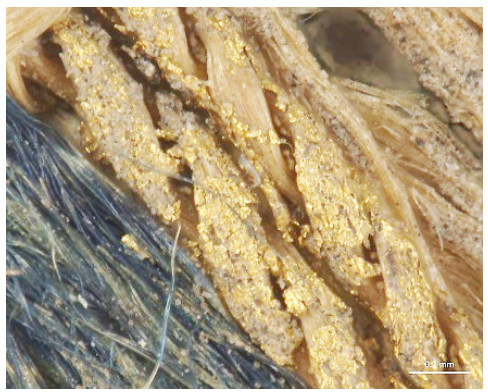

Figure 7. Natural soil on metal foil Figure 8. Natural soil on metal leaf adwithout organic substrate around silk hering directly around silk core $(\times 40)$, core $(\times 80)$, embroidery with tiger-drag- cloth and border fragments, Yuan on, Liao Dynasty, China, 1997.216a, b. Dynasty, China, 1998.114a-d.
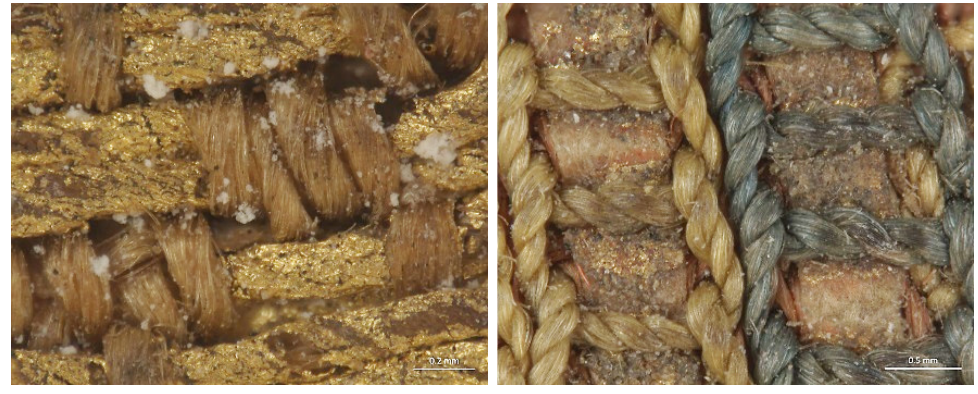

Figure 9. Natural soil on metal leaf ad- Figure 10. Natural soil on metal foil hering directly around silk core $(\times 80)$, with paper substrate $(\times 40)$, decorative cloth and border fragments, Yuan pendant, early 15C, China, 1989.4. Dynasty, China, 1998.114a-d.

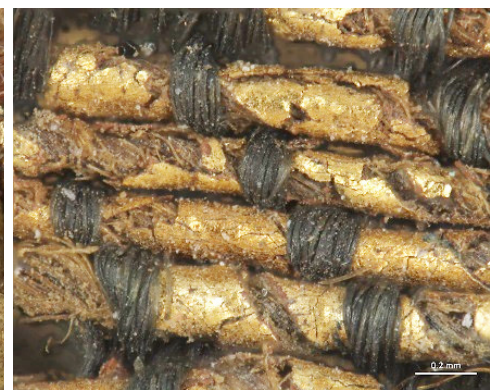

Figure 12. Natural soil on metal foil Figure 13. Natural soil on metal foil

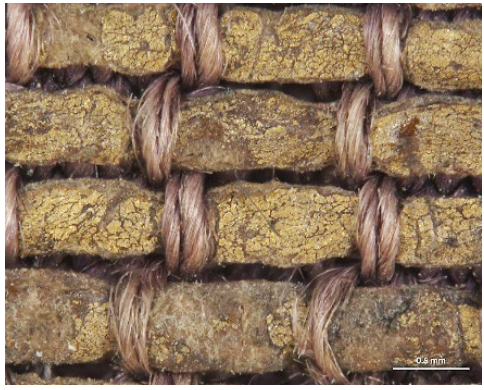

Figure 11. Stain on metal foil with paper substrate $(\times 40)$, textile fragment, 13C-mid 14C, China, 1998.438.
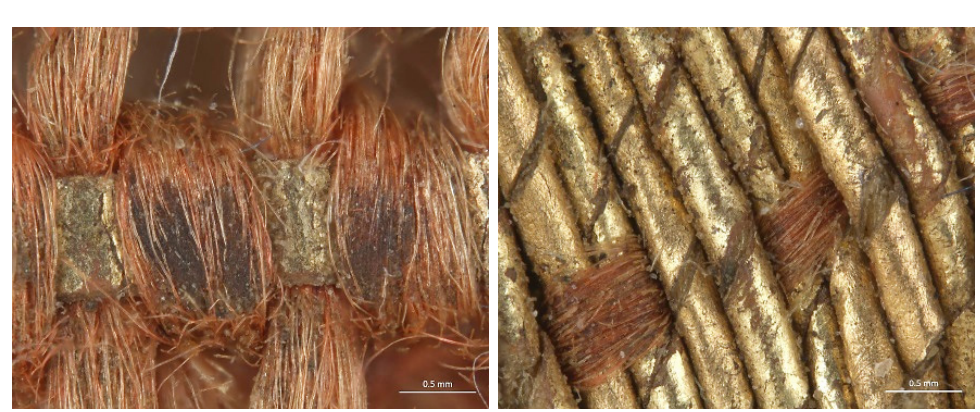
with paper substrate $(\times 40)$, rank badge, 15C, China, 1988.154.1.

with paper substrate around a silk core $(\times 40)$, rank badge, 15C, China, 1988 . 154.1.
Figure 14. Natural soil on metal foil with paper substrate around a silk core $(\times 80)$, woman's dragon robe, Qing Dynasty, Yongzheng Period, China, 62.162. 
metal thread. It showed the brownish-grey color of natural soil composed of fine dust particles penetrating into the fibers and scattered over the surface of the textile and its metal thread(Figures 6,7). The cloth and border fragment, dating to the Yuan dynasty(1271-1368), is reddish-brown silk $\mathrm{Z}$ twisted with gold leaf adhering directly to the wefts, with light greyish-beige colored soil on the surface of metal thread(Figures 8,9).

The decorative pendant is an embroidery with metal thread on silk satin, dating to the Ming dynasty(Figure 10). The textile fragment is a $13^{\text {th }}-14^{\text {th }}$ century woven textile with a pattern of hares; it is identical in pattern, color, and weave structure. Half of the textile was gradually stained to a dark brown color(Figure 11). A rank badge from the Ming dynasty(1368-1644) consists of two different types of metal thread: metal foil with paper substrate and metal foil with paper substrate around a silk core. Dark black colored soil and dirt appear on the surface(Figures 12, 13). A woman's dragon robe was presumably excavated from the tomb of Guo Qin Wang; the design was identical to that on the left so that dragons were not confronted as they appeared on the front of the robe; the observed fading and overall discoloration of yellowish brown were due to burial conditions. Light brownish grey soil and small-sized fiber fragments were investigated on the surface of this textile with metal thread(Figure 14).

\subsection{Cleaning}

3.2.1. Cleaning of metal samples deteriorated by light (UV and daylight)

Color difference can be expressed as a single numerical value, $\triangle E^{*} a b$, which indicates the amount of the color difference in $\mathrm{L}^{*}, \mathrm{a}^{*}$ and $\mathrm{b}^{*}$ space. The color change pertaining to $\triangle E^{*}$ ab value on the fabricated metal samples deteriorated by light factor after cleaning of synthetic soil showed outcomes: the biggest $\triangle E^{*}$ ab values were observed in kneaded rubber cleaning of gold thread as 17.83 and Stoddard solvent cleaning of silver and copper threads with 10.95 and 17.77. Compared with overall effects of mechanical cleaning and solvent cleaning, the wet cleaning methods were not sufficient cleaning synthetic soil on the surface of metal thread. After mechanical cleaning methods of brushing and vacuuming, gold thread deteriorated by light showed an $\triangle E^{*}$ ab value of 7.59 , while silver and copper thread indicated 3.06 and 6.00 of $\triangle E^{*} a b$ respectively. DI water immersion of the gold, silver and copper thread showed $\triangle \mathrm{E}^{*} \mathrm{ab}$ values of $2.33,1.48$, and 3.83 with the result showing that wet cleaning was not effective, and in addition that a rapid humidity change between wet and dry conditions made the edges of samples with paper substrate lose their original shape. $\triangle E^{*} a b$ values after $\mathrm{n}$-decane with those samples showed 5.27, 7.72 , and 14.65 while those of n-hexane were measured as 15.24, 5.74, and 14.21(Figure 15).

In the $\mathrm{L}^{*}, \mathrm{a}^{*}$ and $\mathrm{b}^{*}$ color space, $\mathrm{L}$ indicates lightness and the lighting effect was outstanding in the treated samples with kneaded rubber, Stoddard solvent, n-decane and $\mathrm{n}$-hexane. Both the brushing and vacuuming, and kneaded rubber treatments showed better whitening effects from these cleaning processes than did wet cleaning methods. Brushing and vacuuming could remove big particles. However, pigment or small particles of synthetic

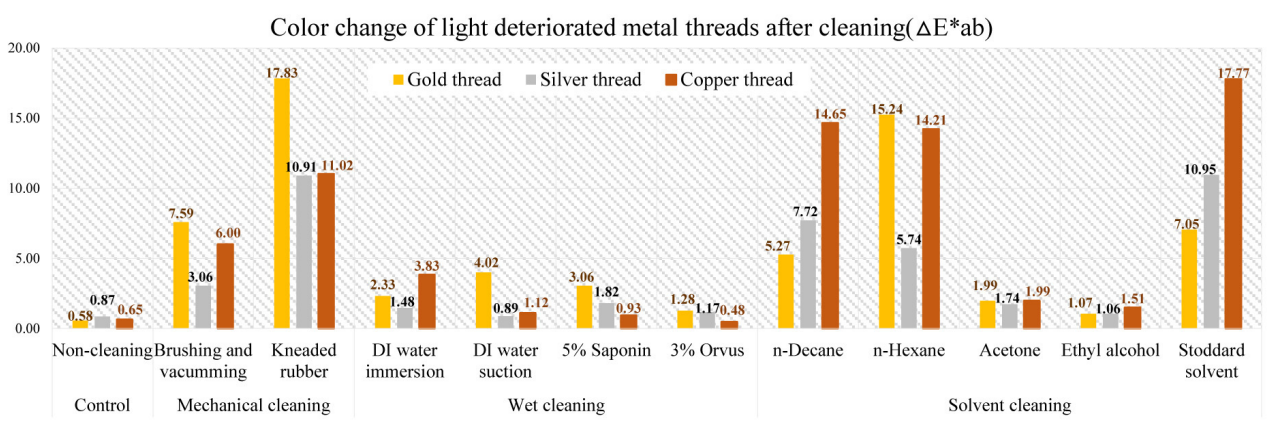

Figure 15. Color change of light deteriorated metal threads after cleaning $\left(\triangle E^{*} a b\right)$. 
soil could not be removed completely. Kneading rubber was successful in picking up both large and small particles, but the stickiness of the particles on the rubber caused some metal leaf peel off after cleaning(Figures 16-18). With the solvent cleaning methods, Stoddard solvent had a good cleaning effect but ethyl alcohol was not effective in

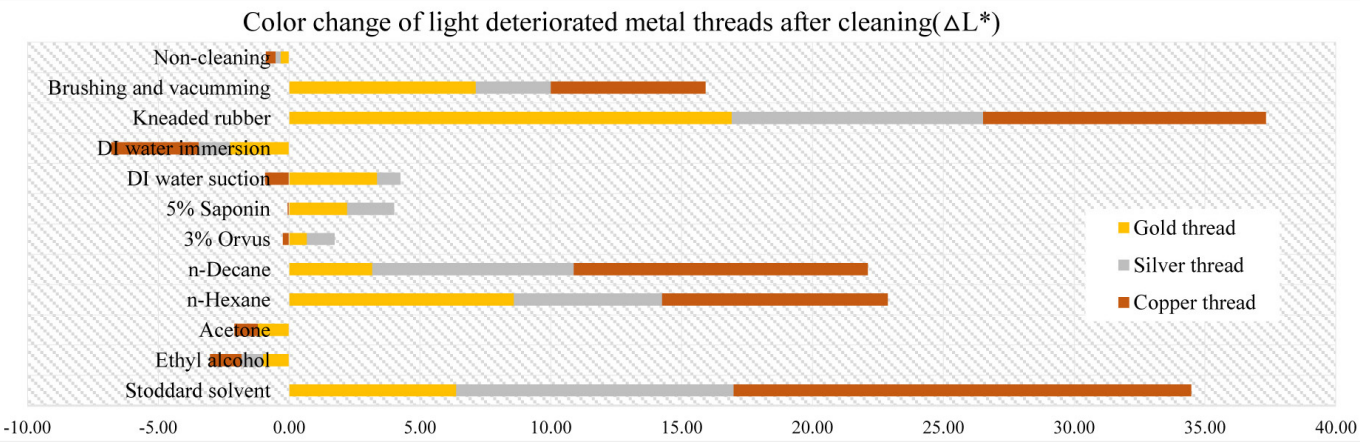

Figure 16. Color change of light deteriorated metal threads after cleaning $\left(\triangle L^{*}\right)$.
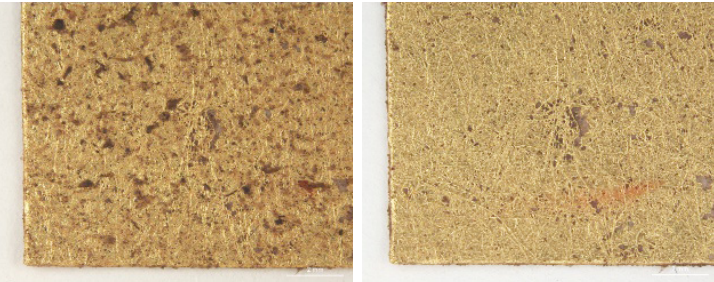

Figure 17. Before and after brushing and vacuuming cleaning of light deteriorated gold thread.
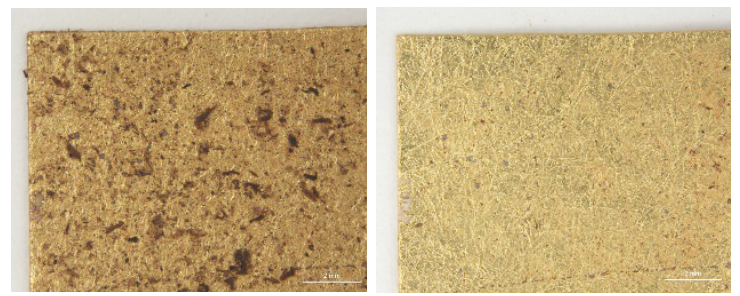

Figure 19. Before and after Stoddard solvent cleaning of Figure 20. Before and after ethyl alcohol cleaning of light light deteriorated gold thread.
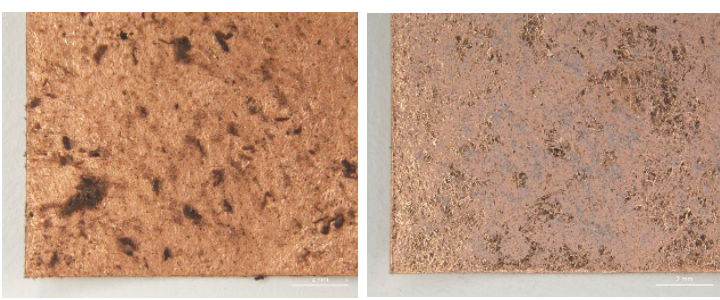

Figure 21. Before and after n-hexane solvent cleaning of Figure 22. Before and after n-decane solvent cleaning of light deteriorated copper thread.
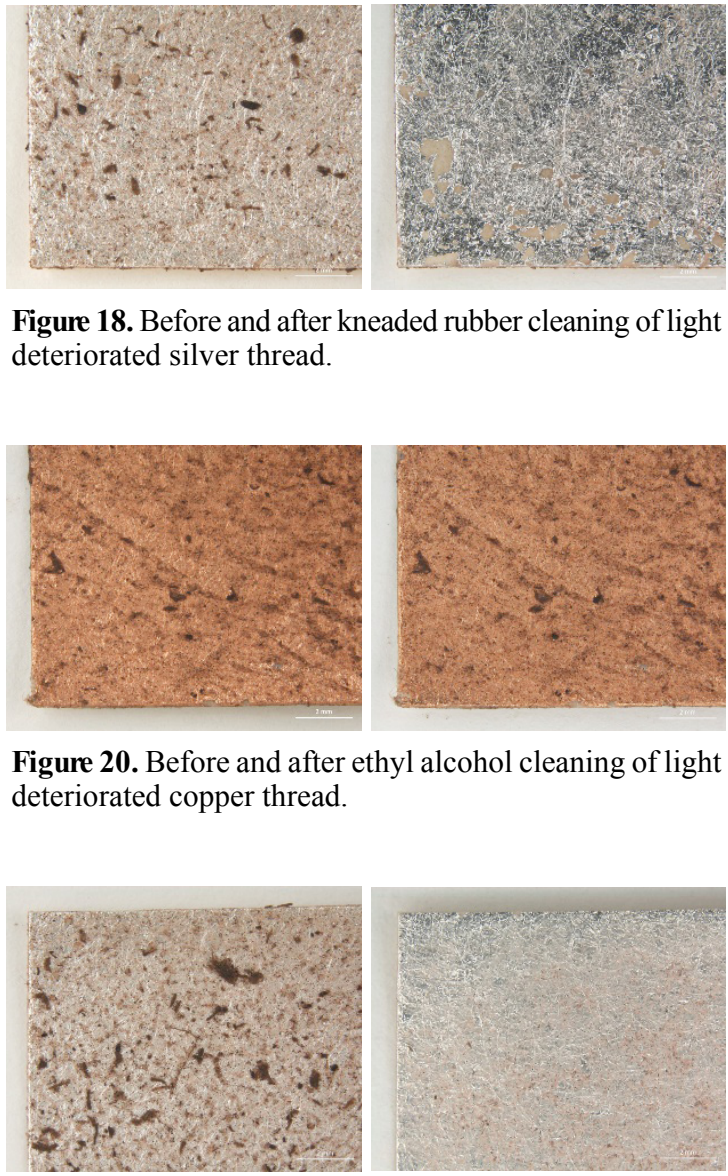

Figure 18. Before and after
deteriorated silver thread. deteriorated copper thread.

light deteriorated silver thread. 
cleaning the synthetic soil. After the n-hexane and n-decane cleaning process, a white layer remained on the samples' surface(Figures 19-22).

\subsubsection{Cleaning of metal samples deteriorated by temperature and humidity}

The color change pertaining to $\triangle E^{*} a b$ values on the cleaning of deteriorated metal samples by temperature and humidity showed the following outcomes: the greatest $\triangle E^{*} a b$ values were seen in the gold thread treated with n-hexane: 25.36. Compared with mechanical and solvent cleaning, the wet cleaning method was not sufficient to remove the synthetic soil from the prepared samples: DI water immersion, DI water suction, saponin, and Orvus. In the mechanical cleaning, $\triangle \mathrm{E}^{*} \mathrm{ab}$ values of gold, silver, and copper thread after brushing and vacuuming were 4.87, 4.85 , and 3.34 while those of kneaded rubber treated samples were measured as 7.57, 9.90, and 11.56; $\triangle \mathrm{E}^{*} \mathrm{ab}$ values of gold, silver, and copper thread after Stoddard solvent cleaning showed color differences of 14.30, 11.44, and 11.06(Figure 23).

Through the investigation on the brightness effect of each cleaning, use of Stoddard solvent showed the most significant brightness effect: the original natural gloss was exposed on the surface after cleaning. Kneaded rubber showed good cleaning effect by picking up the synthetic soil particles, but the metal leaf peeled off because of the rubber's adhesive qualities. Wet cleaning methods, including DI water immersion, DI water suction, 5\% saponin and 3\% Orvus, did not show a good cleaning effect while solvent cleaning with n-decane and n-hexane caused the white opaque layers(Figures 24-28).

\section{CONCLUSION}

On the naturally-soiled textiles, the colors and particle sizes of soil materials were diverse due to the different circumstances of their original environmental factors and

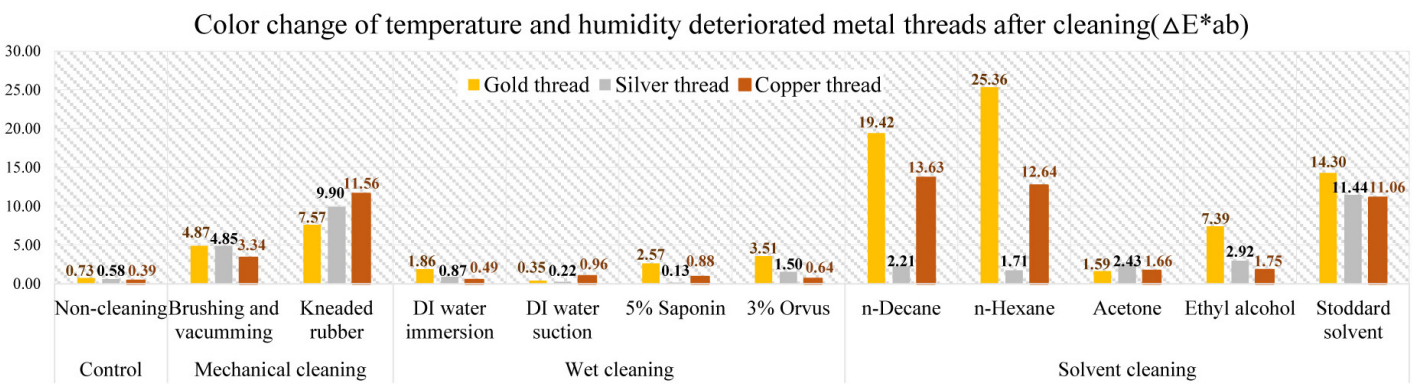

Figure 23. Color change of temperature and humidity deteriorated metal threads after cleaning $\left(\triangle \mathrm{E}^{*} \mathrm{ab}\right)$.

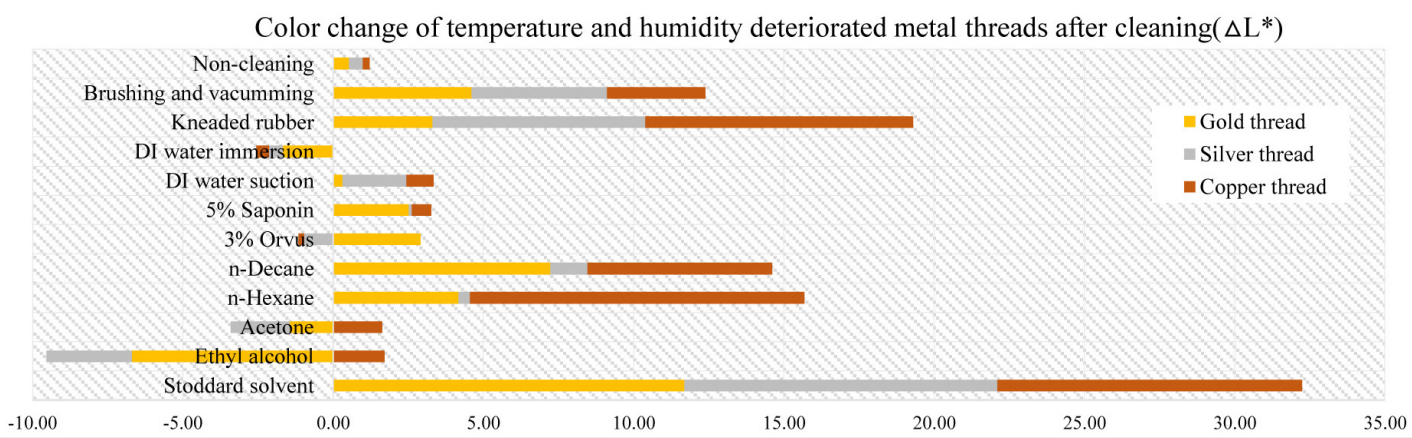

Figure 24. Color change of temperature and humidity deteriorated metal threads after cleaning $\left(\triangle L^{*}\right)$. 
surface conditions. Different discoloration of naturallyaccumulated soil on the textiles ranged from light beige, brownish grey, dark brown and black, and yellowish brown. Particle sizes and textures of each different soil covered the six soiled objects with metal thread were diverse and included small pieces of textile fiber fragments on their surfaces. Prior to the cleaning process, deterioration with light(UV and daylight), and temperature and humidity was conducted with fabricated gold, silver, and copper threads in the laboratory. However, there were insufficient amounts of soils and stains sitting on the surface due to the controlled environment and limited external material sources during the artificial deterioration process, unlike historic and/or archaeological textiles with metal thread (Park et al, 2018).

We added synthetic soil to the surface of the samples which had been deteriorated by light(UV and daylight), and temperature/humidity. The synthetic soiling mixture consisted of carbon black, iron oxide, gelatin, soluble starch, cement, silica, lime, kaolin, peat moss, and mineral oil. During the application of synthetic soil, the dry soiling mixture was found to be difficult to apply evenly over the samples' surface; however, we soon discovered that a $12.1 \mathrm{~g}$ drying mixture with $20 \mathrm{~mL}$ solution of $4.5 \%$ mineral oil in chloroform (w/w) created a slurry texture to be applied to the surface of metal samples. Next, 11 different types of textile cleaning methods were used to clean the synthetic soil sitting on the deteriorated metal thread.

Mechanical cleaning methods were used: brushing and vacuuming methods, and kneaded rubber. Brushing and vacuuming is known as one of the first step of cleaning and many cleaning processes of kneaded rubber are used in common; kneaded rubber is composed of natural and/or synthetic rubber, vulcanized vegetable oil, antioxidants, mineral oil, pumice, calcium carbonate, titanium dioxide, and carbon black(Estabrook, 1989). Among both light and temperature and humidity deteriorated samples, overall color changes with $\triangle E^{*}$ ab values after the uses of kneaded rubber were greater than those treated with brushing and vacuuming. The $\triangle E^{*} a b$ value of light deteriorated gold treated with brushing and vacuuming was 7.59 and that of kneaded rubber was $17.83 ; \triangle \mathrm{E}^{*} \mathrm{ab}$ values of temperature and humidity deteriorated gold after brushing and vacuuming, and kneaded rubber were measured as 4.87 and 7.57. Brushing and vacuuming can remove the large-size particles but fine pigment or small particles of synthetic soil could not be removed completely; kneaded rubber was
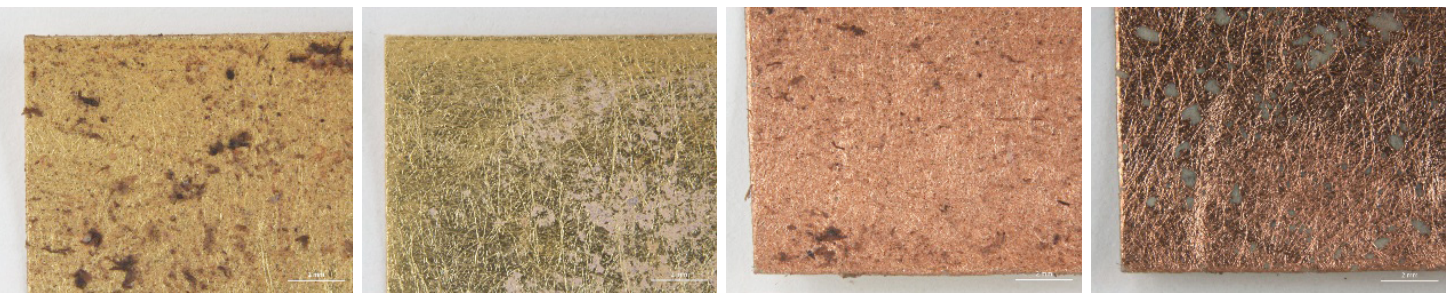

Figure 25. Before and after Stoddard solvent cleaning of Figure 26. Before and after kneaded rubber cleaning of temtemperature and humidity deteriorated gold thread. perature and humidity deteriorated copper thread.
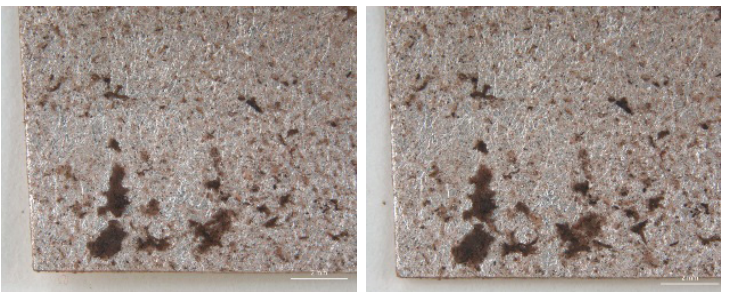

Figure 27. Before and after DI water immersion of temperature and humidity deteriorated silver thread.
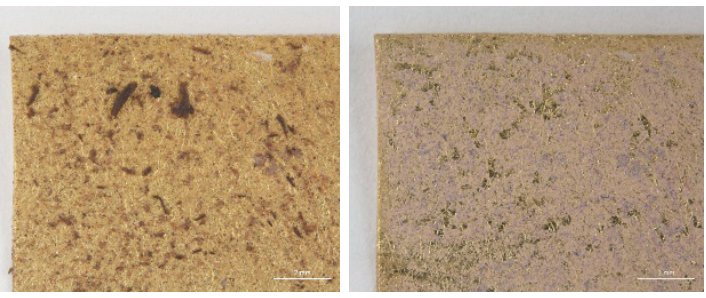

Figure 28. Before and after n-decane of temperature and humidity deteriorated gold thread. 
successful in picking up large and small particles but its tackiness weakened some metal leaf and caused it to peel off after cleaning. This result coincides with a previous report that the attempt to remove with a pencil eraser even with great caution, was unsuccessful because the rubber eraser damaged the silk threads securing the thin metal wire(Toth, 2012).

Second was the wet cleaning with DI water immersion, DI water with suction table, $5 \%$ saponin, and 3\% Orvus WA Paste. Wet cleaning has been widely used as the textile conservation treatments using water alone as a solvent, and as a solvent and/or medium for surface-active and anti-soil agents or additives. In this study, DI water was applied alone with two methods: aggressive immersion and gentle soft penetration under the suction table. DI water immersion of light deteriorated gold, silver and copper thread showed $\triangle E^{*}$ ab values of $2.33,1.48$, and 3.83 ; those of temperature and humidity deteriorated samples were measured as $\triangle \mathrm{E}^{*} \mathrm{ab}$ values of $1.86,0.87$, and 0.49 . Wet cleaning was not effective and the rapid humidity changes between wet and dry conditions caused the edges of the paper substrate to lose their original shape.

Saponin is an extraction material made from ginseng and beans. It is part of a large family of natural detergents with structurally related compounds of steroid or triterpenoid aglycone, linked to one or more oligosaccharide moieties by glycosidic linkage. Its physiochemical and biological properties with both polar(sugar) and nonpolar groups(steroid and/or triterpene) have led to traditional and industrial applications with strong surface-active properties to liquid detergents and a long-lasting foaming agent(Oakenfull, 1981; Price et al, 1987; Martin and Briones, 1999; Makkar et al, 2007; Chen et al, 2010). Orvus WA Paste is near-neutral $\mathrm{pH}$ with excellent detergency, anionic synthetic surfactant and wetting agent with sodium dodecyl/lauryl sulphate. It is biodegradable and soluble in both hot and cold water, lowering surface tension and providing fast wetting and penetration. Wentz and Tímár-Balázsy reported that Orvus WA Paste does not cause significant color change and staining with tested natural colorants(Wentz, 1986; Tímár-Balázsy, 2000). However, after wet cleaning with saponin and Orvus, synthetic soils were not removed and cleaning efficiency with wet cleaning was not sufficient to clean off the synthetic soil.

Third was solvent cleaning with n-decane, n-hexane, aceton, ethyl alcohol and Stoddard solvent. Stoddard solvent is a distilled petroleum mixture of $\mathrm{C}_{7}-\mathrm{C}_{12}$ hydrocarbons, a dry cleaning agent; its mixture consists of three major groups of component linear and branched alkanes, also known as branched chain alkancese, cycloalkanes, and aromatic hydrocarbons(McDermott, 1975). After cleaning with Stoddard solvent, $\triangle E^{*}$ ab values of gold thread with both light deterioration and temperature and humidity deterioration were measured as 7.05 and 14.30; those of silver thread were 10.95 and 11.44 , and those of copper thread were observed as 17.77 and 11.06. The lightening effect of deteriorated samples was significant with Stoddard solvent, n-decane, and n-hexane though white opaque layers remained on the cleaned metal thread.

Through the overall investigation of the brightness effect of each cleaning, Stoddard solvent cleaning showed the most significant brightness effect as the original natural gloss became visible on the surface after cleaning; kneaded rubber showed effective cleaning by picking up the synthetic soil particles. However, the metal leaf peeled off because of its tackiness. In the wet cleaning methods, including the method with DI water immersion, DI water suction, 5\% saponin and 3\% Orvus, a good cleaning effect was not shown, while solvent cleaning with n-decane and n-hexane caused the white opaque stains on the surface after cleaning.

It can be predicted that historic and archaeological metal thread has been deteriorated by complex environmental factors for a long time, and that naturally occurring soil is more likely to contain more complicated materials and sources than the synthetic soil applied in this study. Hence, an application with naturally-soiled textile samples with metal thread is needed to assess the cleaning efficiency and stability for the following experiments. In addition, further cleaning studies which use more than two cleaning methods together are needed. Moreover, such experiments should 
include different cleaning agents, sophisticated cleaning techniques, and other deteriorating environment factors, additional fabrication processes, and materials including various metal alloys and types of adhesive and paper. The success of cleaning in terms of both aesthetic effect and preservation of the integrity of textiles and their metal threads depends on the skill and methods employed by textile conservators and specialists.

\section{ACKNOWLEDGEMENTS}

Our thanks must go to the "Academic \& Professional Program, Andrew W. Mellon Fellowship in the Department of Textile Conservation at The Metropolitan Museum of Art, New York(2017-2018)" and "Specialized Studies Program from the Department of Heritage Conservation \& Restoration, Graduate School of Cultural Heritage, Korea National University of Cultural Heritage(2017)" for the valuable opportunity and experience in New York. We also would like to especially thank the department head, Janina Poskrobko and every staff members in the Department of Textile Conservation, the Department of Scientific Research, the Department of Paper Conservation, the Department of Asian Art, Antonio Ratti Textile Center, Thomas J. Watson Library and Education Department at the Metropolitan Museum of Art, New York.

\section{REFERENCES}

Ahmed, H.E., 2014, A new approach to the conservation of metallic embroidery threads in historic textile objects from private collections. International Journal of Conservation Science, 5(1), 21-34.

Chen, Y.F., Yang, C.H., Chang, M.S., Ciou, Y.P. and Huang, Y.C., 2010, Foam properties and detergent abilities of the saponins from camellia oleifero. International Journal of Molecular Science, 11, 4417-4425.

Chi, Y.S. and Obendorf, S.K., 1998, Aging of oily soils on textile materials: a literature review. Journal of Surfactants and Detergents, 1(3), 407-418.

Estabrook, E., 1989, Considerations of the effect of erasers on cotton fabric. Journal of the American Institute for Conservation, 28(2), 79-96.

Makkar, H.P.S., Siddhuraju, P. and Becker, K., 2007, Plant secondary metabolities : methods in molecular biology. Humana Press, New York, 93-100.

Martin, R.S. and Briones, R., 1999, Industrial uses and sustainable supply of Quillaja saponaria (Rosaceae) saponins. Economic Botany, 53(3), 302-311.

McDermott, H.J., 1975, Stoddard solvent (mineral spirits, white spirits). American Industrial Hygiene Association Journal, 36(7), 553-558.

Oakenfull, D., 1981, Saponins in food - a review. Food Chemistry, 7(1), 19-40.

Park, H.J., Hwang, M. and Chung, Y.J., 2018, Metal thread: an assessment of the deterioration of metal thread with light, and temperature and humidity factors - a study of textile collections from Korea, China, Japan, and Central Asia and fabricated metal thread at the Metropolitan Museum of Art, New York -. Journal of Conservation Science, 34(4), 129-135.

Price, K.R., Johnson, I.T. and Fenwick, G.R., 1987, The chemistry and biological significance of saponins in foods and feeding stuffs. Journal CRC Critical Reviews in Food Science and Nutrition, 26(1), 127-135.

Que Hee, S.S., Peace, B., Clark, S., Boyle, J.R., Bornschein, R.L. and Hammond, P.B., 1985, Evolution of efficient methods to sample lead sources, such as house dust and hand dust, in the homes of children. Environmental Research, 38(1), 77-98.

Ree, W.H., 1962, A study of the optics of soiling of textile materials using artificial-soil techniques. Journal of the Textile Institute, 53(5), 230-249.

Reynolds, S.J., Etre, L., Thorne, P.S., Whitten, P., Selim, M. and Popendorf, W.J., 1997, Laboratory comparison of vacuum, OSHA, and HUD sampling methods for lead in household dust. American Industrial Hygiene Association Journal, 58(6), 439-446.

Roger, D.L., Patrick, N.B., David, A.S. and Brad, K., 1999, A soiling system for evaluation of house dust, allergens, and lead retention on carpets and other surfaces. Applied Ouccupational and Environmental Hygiene, 14(12), 
$845-851$

Tímár-Balázsy, A., 2000, Wet cleaning of historical textiles : surfactants and other wash bath additives. Studies in Conservation, 45(1), 46-64.

Toth, M., 2012, Lessons learned from conserving metal thread embroidery in the Esterházy collection, Budapest, Hungary. Studies in Conservation, 57(1), 305-312.
Wentz, M., 1986, Historic textile and paper materials : conservation and characterization. American Chemical Society, Washington, DC, 211-230.

Wolbers, R.C., 1992, The use of a synthetic soiling mixture as a means for evaluating the efficacy of aqueous cleaning materials on painted surfaces. Conservation-Restauration des Biens Culturels, 4, 22-26. 